\title{
Una nueva pedagogía dentro del paradigma humanístico para la enseñanza de una segunda lengua ${ }^{1}$
}

\section{Olga Chaves Carballo}

Universidad Nacional, Costa Rica

\section{RESUMEN}

Se propone una nueva pedagogía para la construcción de conocimiento dentro del nuevo paradigma humanístico. Esta pedagogía procura formar a una persona capaz de autocrearse y construir conocimiento que le permita desarrollar sus habilidades lingüísticas. Para poner en práctica esta nueva pedagogía. el mediador debe facilitar el proceso de autoorganización del conocimiento y propiciar el diálogo por medio de experiencias de aprendizaje amenas y gozosas.

\section{Abstract}

A new pedagogy is proposed for the construction of knowledge within a humanistic paradigm. The intention of this pedagogy is to form an individual capable of self-creation and constructing knowledge that allows him/ her to develop linguistic skills. To implement this new pedagogy. the mediator must facilitate the process of the self-organization of knowledge and provide for dialogue through enjoyable and entertaining learning experiences.

Artículo basado en una ponencia presentada en el I Congreso Internacional de Lingüística Aplicada. llevado a cabo en octubre de 2007. en el Campus Omar Dengo. de la Universidad Nacional de Costa Rica.

ᄂ Correo electrónico: profeolgachaves@ hotmail.com 
Palabras clave: enseñanza de segundas lenguas, pedagogía, paradigma holístico, aprendizaje significativo

Keywords: second-language teaching, pedagogy, holistic paradigm, leaming experiences

El paradigma humanístico promueve una nueva pedagogía para la construcción del conocimiento que les da preeminencia a los papeles del aprendiente ${ }^{3}$, mediador ${ }^{4}$ y ambiente pedagógico con experiencias de aprendizaje significativas y gozosas en la enseñanza de una segunda lengua. Esta nueva pedagogía propicia el reencantamiento de la educación, ya que incorpora a la formación de los aprendientes los siguientes fundamentos: $a$. la creatividad y el pensamiento crítico: para actuar en procura de la realización personal, la comprensión del ser humano y la construcción social; $b$. la visión integral de la vida: el pensamiento integral es un pensamiento de procesos, los cuales pueden cambiar y autorenovarse; $c$. el principio de autoorganización: la autonomía que permite resolver problemas, tomar decisiones y realizar acciones en pro del bienestar humano.

Para ello, se requiere un cambio de conciencia que deje atrás el paradigma cartesiano o mecanicista, en el cual los sistemas educativos se caracterizan por la departamentalización, las disciplinas. los horarios, la desintegración del saber y la desmotivación estudiantil. Ante la inquietud por la deserción de los aprendientes de las aulas tradicionales, la falta de pensamiento crítico y la carencia de innovación en el proceso educativo, los educadores pedagogos han propuesto una pedagogía cuyo objetivo es inspirar al aprendiente y al mediador para que vivan el proceso de aprendizaje desde sus propios intereses y creencias, mediante experiencias que los motiven de manera intrínseca y armoniosa. Esta visión requiere una transformación de conciencia que permita adaptarse al cambio; ya que como dicen Briggs y Peat

\footnotetext{
Se refiere al estudiante en el nuevo paradigma.

+ Se refiere al profesor.
} 
«... ese 'yo', ... en nuestro centro psicológico, está sometido al cambio constante» 5 .

Para los educadores que concuerdan con la reimplantación de la educación como un sistema integral de valores y opciones, esta toma de decisiones es viable y no idealista debido a que somos nosotros mismos quienes iniciamos la misión; somos los pioneros que crean las posibilidades para que esta propuesta se lleve a cabo y se inicie la reconquista de aprendientes, para formarlos dentro del modelo pedagógico propuesto. Los educadores formados en este nuevo paradigma, apoyamos en las aulas de enseñanza de idiomas a los estudiantes que crean, participan y presentan diferentes actividades, como debates, diálogos, obras de teatro, canciones, poesía, discusiones grupales, presentaciones de situaciones reales y muchas más que les ayudan a desarrollar sus destrezas lingüísticas; los vemos disfrutar del proceso enseñanza-aprendizaje porque se identifican con la pedagogía que los incentivan a construir, expresar y razonar. Incitamos «a los jóvenes a mirar y a escuchar, a superar lo que se da ya por sentado y lo rutinario» ${ }^{6}$. Así, logran despertar y empezar a vivir, junto a otras personas, reconstruyendo sus propios mundos hacia la libertad, la autenticidad y la dignidad para el desarrollo de su verdadera naturaleza.

\section{Un cambio de paradigma}

Actualmente, estamos en un mundo en el que la realidad se transforma diariamente; esto exige nuevas modalidades y concepciones que emergen de una educación más flexible, con el fin de satisfacer las necesidades y los retos de los estudiantes en el siglo xxI. Con la visión de un nuevo paradigma, el aprendizaje se construye con base en momentos creativos y de reflexión y en cambios o transformación de conciencia para hacer de la vida un continuo aprender.

John Briggs y David Peat, Las siete leyes del caos (Barcelona: Dimas Mas. 1999) 5.

( Maxine Greene. Liberar la imaginación (Barcelona: Graó. 2005) 62. 
Asimismo, la humanidad ha iniciado una nueva etapa que rompe con una vieja práctica de fragmentación la cual, a su vez, ha causado falta de motivación escolar y deseo de encontrar en las aulas las respuestas a la existencia. Entonces, los educadores adoptamos la visión holista como acto transformador, una perspectiva que la cual «se basa en la certidumbre de que todo está interconectado: Estamos unidos a los demás y a las formas no humanas que nos rodean» ${ }^{7}$. El holismo es una visión que nos une y un modo de vivir que trae libertad, espiritualidad y humanidad. En esta dimensión, todo nuestro ser interviene en el proceso de aprendizaje; somos seres integrales con libertad de recrear y hacer del proceso de aprendizaje un disfrute. De esta forma, la mediación pedagógica propone la creación de experiencias de aprendizaje agradables, significativas y novedosas. Así, la mente holista permite tanto la construcción del conocimiento, como la expresión de sentimientos, motivaciones y emociones.

A partir de los planteamientos del paradigma holístico, se aprende de una manera gozosa y placentera, lo cual facilita la adquisición de una segunda lengua. El estudiante se siente parte del ambiente de clase, desarrolla relaciones sociales con sus compañeros, se motiva para participar en las actividades de trabajo que propicien el aprendizaje del uso del idioma y mantiene una actitud positiva hacia la meta deseada.

En esta pedagogía, además, se procura aprender por medio del placer, lo cual se refiere a sentirse a gusto y accionar la apertura de pensamiento para entender, compartir y apropiarse del conocimiento. Assmann señala que «el conocimiento sólo emerge en su dimensión vitalizadora cuando tiene algún tipo de vinculación con el placer» ${ }^{8}$. Asimismo, Wagensberg afirma que sin gozo no hay pedagogía y que no hay conocimiento verdadero sin disfrute; su obra El gozo intelectual es una celebración del placer del aprendizaje que nos proporciona

\footnotetext{
7 Ramón Gallegos, Educación holista: pedagogía del amor universal (México. DF: BECA. 1999) 28

8 Hugo Assmann, Placer y ternura en la educación: hacia una sociedad aprendiente (Madrid: Nancea. 2002) 28.
} 
nuestro complejo cerebro. Un gozo intelectual que, además de deleitarnos y mantener activas nuestras neuronas, nos mantiene más vivos. Los tres pasos para el gozo intelectual son el estímulo, la conversación y la comprensión. El estímulo es el motor de la curiosidad, el cual da paso a la conversación, la cual a la vez permite la comprensión que se traduce en gozo ${ }^{9}$. De esta forma, se pretende despertar en los aprendientes el diálogo, el entendimiento y la apertura de pensamiento para accionar ante situaciones relacionadas con sus intereses y con los de los demás.

Gallegos opina que esta visión holística busca formar seres humanos responsables, que honren la verdad, la bondad y la belleza de una vida en la cual la espiritualidad sea la esencia de lo que somos, nuestra verdadera naturaleza, el último contexto de significado de nuestras vidas ${ }^{10}$. El aprendiente se transforma en un ser orientado a aprender, para llegar a ser un individuo que se preocupa por su forma de vivir y considera la relación con su entorno, el cual lo nutre de energía vital.

\section{La nueva pedagogía}

En la pedagogía liberadora del nuevo paradigma se integra la educación de los idiomas en forma de experiencias significativas y dignas de realización. Esta pedagogía aprecia a los aprendientes como sujetos de aprendizaje activo; los convierte en personas curiosas y críticas que abren sus propios mundos a la reflexión, al descubrimiento y a la transformación de nuevas dimensiones. Además, enseña estralegias para el desarrollo del conocimiento y de las experiencias que necesitan para enseñarse a sí mismos; "para imaginar, para elegir, y actuar desde sus propios ángulos de percepción de posibilidades» ${ }^{11}$.

Jorge Wagensberb. El gozo intelectual (Barcelona: Tusquets, 2007); <www.hoy,es/prensa200070610/ sociedad/gozo-intelectual-libro-sobre_200070610.html>.

11. Ramón Gallegos. El espíritu de la e educación: hacia una sociedad aprendiente (Madrid: Narcea, 2004).

11 Greene. 70. 
Igualmente, es una propuesta para la promoción del aprendizaje grupal en un ambiente que propicie las relaciones e interrelaciones armoniosas que contribuyan a una comunicación más abierta, al trabajo cooperativo y la convivencia. En esta pedagogía, el individuo decide qué lo motiva, lo atrae y lo mantiene enérgico para convivir e interactuar en grupo; busca la comprensión de conceptos y aporta a la construcción de conocimientos. De tal manera, esta actitud podrá ayudar a cambiar y sanar el mundo, a vivir en plenitud y volver a encantarse con la vida. Greene nos invita a reflexionar y afirma: «No debemos dejar pasar el tiempo sin buscar nuevas conmociones de conciencia, nuevas exploraciones, nuevas aventuras del significado, nuevas formas activas e incómodas de participar en la búsqueda interminable de la comunidad humana» ${ }^{12}$.

Esta pedagogía crea espacios de disfrute y de complacencia para iluminar el sendero del quehacer y el ser y percibir nuevos órdenes de estructuras, ya que la educación tiene el fin de educar para la vida, los educadores nos comprometemos a encontrar alternativas pedagógicas que fomenten el desarrollo del conocimiento para aprender a vivir en un mundo mejor, porque al fin y al cabo, esta es «una búsqueda de la visión social futura de una comunidad más humana, más plenamente pluralista, más justa y más feliz» ${ }^{13}$.

Con estos espacios creados, junto al despertar de una sensibilidad, de la reflexión y el diálogo, se generan oportunidades para aprender, construir y fomentar nuevas ideas. Asimismo, estas oportunidades mejora su autoestima, una revelación del mundo por medio de los sentidos que no permite coartar la creatividad; por lo contrario, da libertad, emoción y deseo de descubrir y crear.

Esta nueva pedagogía permite:

- Una formación humana: el aprendiente decide vivir con sentido y significado y acepta todos los potenciales y modos de aprender.

\footnotetext{
Greene. 232.

Greene. 101.
} 
- Reconocimiento de la espiritualidad en los aprendientes: no solo son poseedores de conocimientos, sino que también son seres capaces de desarrollar una inteligencia espiritual que les permite ensanchar los límites de un aprendizaje flexible, visionario y creativamente espontáneo.

- Atención a la diversidad: todos son diferentes, aprenden de manera particular y enfrentan el mundo con su propia visión.

- Cambios de conciencia: el aprendiente es un ente de cambio, una persona que quiere buscar soluciones a los problemas del mundo; siente la necesidad de encontrar respuestas a sus inquietudes y de trascender para reconstruir su realidad.

\section{$\Lambda$ prendiente: creador de su aprendizaje}

Se considera al aprendiente como una persona capaz de optimizar sus canales de percepción y de redescubrir cuán reconfortante resulta aprender con emoción. Su creatividad lo adapta para experimentar, investigar, construir y apropiarse de los conocimientos, con el fin de alcanzar su autorrealización. Como afirma Morin, «la mente humana se abre al mundo. La apertura al mundo se revela por la curiosidad, la interrogación, la exploración, la búsqueda, y la pasión de conocer» ${ }^{14}$.

Como sistema vivo, el aprendiente posee la capacidad de autoorganizarse, además puede inventarse y reinventarse constantemente. Ha pasado de pertenecer a un paradigma tradicional en el cual las acciones estaba mecánicamente predeterminado, fijo y lineal, a formar parte de uno mucho más abierto, flexible y holístico que exige una transformación fundamental del pensamiento, de los valores y percepciones, para convertirse en un ser natural, espiritual y racional, que busca fortalecer sus destrezas lingüísticas para poder comunicarse exitosamente en una segunda lengua.

El aprendiente responde a la naturaleza humana, a esa necesidad natural de descubrir qué lo motiva a aprender una segunda lengua. Es,

\footnotetext{
It Edgar Morin, El método V: la humanidad de la humanidad (Madrid: Cátedra. 2003) 44-45.
} 
al mismo tiempo, cuerpo y espíritu: sentimientos, emociones, necesidades, potencialidades, intereses y expectativas; es incluso capaz de trascender la realidad que lo envuelve. Morin dice que este aprendiente «aspira a vivir plenamente su vida, con felicidad, amor, bienestar, acción, contemplación, conocimiento, poder, aventura...» 15 .

El proceso de cambio en el aprendiente se da primeramente por medio de experiencias holísticas del aprendizaje, las cuales abren su corazón y le permiten recibir con disfrute lo que le apasiona saber y aprender. Cuando el aprendiente ama el aprendizaje de un idioma, los factores afectivos contribuyen a su adquisición y aprendizaje de una manera más natural y amena. El aprendiente, en un ambiente de gozo, se siente vivo, más creativo; genera respuestas originales, se divierte e inventa soluciones posibles a sus expectaciones.

\section{El mediador: ente de cambio}

El mediador logra promocionar el aprendizaje de una manera creativa, abre espacios para la expresión en todos los ámbitos y promueve la construcción del conocimiento y de experiencias que necesitan los aprendientes para enseñarse a sí mismos. También. el mediador contribuye a cambiar o transformar sus mundos porque crea espacios de «oportunidades abiertas, de posibilidades, de moverse en la búsqueda en pos de algo» ${ }^{16}$.

La interacción del mediador con el aprendiente le permite al primero compartir experiencias con el aprendiente, apoyarlo y asesorarlo en su proceso de aprendizaje por medio de un estímulo en su capacidad para adquirir conocimiento, de retos para que incremente sus saberes y la creación de su propio paradigma en el cual pueda apropiarse de sus conocimientos para actuar desde sus propios ángulos de percepción de posibilidades, para compartirlos y así crecer para su propio desarrollo personal y profesional.

\footnotetext{
Morin. 59.

16 Greene. 70.
} 
El mediador holista incentiva el aprendizaje para el desarrollo humano. El nuevo papel del educador es educar para una participación democrática, educar para promover ciudadanos globales, para una cultura ecológica planetaria y para la espiritualidad. El educador holista nutre el potencial humano, desarrolla el pensamiento creativo, integra el conocimiento, en fin, es creador de un ambiente para la formación integral del ser humano de modo que se pueda vivir responsablemente en una cultura sostenible. Promueve aprender a vivir, a ser, a ver, a desarrollar todos los sentidos con emoción, con ánimo y amor. En las palabras de Gallegos, «...la naturaleza de la conciencia del educador es lo más importante en su formación. A esta conciencia la podemos llamar presencia plena. La presencia plena es el estado natural de la mente, es la presencia en la experiencia de todo lo que somos. la presencia completa del cuerpo, la mente y la conciencia, es un estado de alerta total» ${ }^{17}$.

El mediador holista «puede también sentir un fuerte impulso al abrir caminos que conduzcan mejores formas de enseñanza y de vida ${ }^{18}$, porque promueve la curiosidad por el conocimiento con el fin de que el aprendiente indague, descubra y explore. Es necesario entonces amar el aprendizaje, de una forma que el mediador interesado por aprender se convierte en un agente multiplicador de saberes. Desde esta perspectiva, el aprendiente aprende utilizando los sentidos en ambientes preparados para el gozo. En la mediación pedagógica, por lo tanto, debe tomarse en cuenta el estilo de aprendizaje, el estilo cognitivo propio de cada aprendiente y el modo como se vincula con los otros a partir de sus experiencias previas, en las cuales entran en juego la forma de expresarse y de expresar sus sentimientos, pensamientos, su historia de vida, su presente y las expectativas de su futuro, es decir, todo su ser. «Es por la pasión, por la comunicación, por la relación humana, por la aventura de realizarse como persona, a partir

Gallegos. Educación.... 233.

Greene. 73. 
de la construcción de conocimientos, de la creatividad, de la investigación, del intercámbio de experiencia» ${ }^{19}$.

\section{Ambiente holístico}

En un ambiente holístico las experiencias de aprendizaje creativas, imaginativas y originales son excelentes opciones que le permiten al ser autocrearse y encontrar la satisfacción en el cumplimiento de sus metas y sueños gracias a que, como individuo íntegro, aprende a mostrar respeto hacia las diferencias, preocupación por lo que sucede a su alrededor y amor así mismo y a los demás. Este ambiente debe estar lleno de entusiasmo y energía para que propicie el proceso de construcción de conocimientos. En este aspecto, se respetan los estilos de aprender de cada persona, la forma en que ven la vida, las experiencias previas, sus necesidades, angustias, anhelos y pasiones.

Por otra parte, el aprendizaje debe ser novedoso, dinámico y estimulante para el aprendiente. Como lo afirma Assmann, «aprender es siempre descubrimiento de lo nuevo; si no, no es aprender» ${ }^{20}$. El aprendizaje desde este nuevo paradigma es un medio para alcanzar la autorrealización de todo aquel que expresa libremente sus pensamientos, acorde con su creatividad, originalidad y espíritu-mente. En este escenario, el mediador propicia espacios de interrelación, intercambios de conocimientos de diálogo y de apertura, en los cuales ambos son partícipes activos del proceso pedagógico.

Para desarrollar y promover una enseñanza activa y un aprendizaje significativo para el aprendiente, se debe partir de los siguientes aspectos:

- Propiciar experiencias de aprendizaje que motiven e interesen al estudiante y le permitan enfrentarse a la resolución de problemas, incrementar su creatividad, así como a investigar y construir conocimiento.

19 Francisco Gutiérrez, Biopedagogía (Heredia: Editorialpec, 2003) 50.

20) Assmann. 62. 
- Partir del entorno del aprendiente para identificar sus intereses, actitudes y aptitudes. Así, es posible crear materiales y actividades en el proceso de aprendizaje que llenen sus necesidades de comunicación.

- Fomentar experiencias de auto-evaluación, con respecto a su propio quehacer y adquirir un compromiso con su proceso educativo, desarrollando hábitos de estudio diario, utilizando multimedia y recursos de audio para desarrollar sus destrezas lingüísticas.

El diseño curricular de los cursos del inglés debe estar dirigido il estudiante, como centro del proceso enseñanza-aprendizaje. Dentro de sus fundamentos filosóficos propone el enfoque constructivista, cl cual promueve la mediación del docente en los ámbitos conceptual, metodológico y de evaluación. Se parte de un proceso de enseñanzaaprendizaje continuo y progresivo, en el cual el individuo aprende de manera significativa y permanente al ir construyendo en forma activa sus propios conocimientos, los cuales se alcanzan en diferentes etapas, le manera paulatina.

Se aprende por los actos de autoorganización del conocimiento yuc conducen a la autoconstrucción del ser humano, tales como:

- Visualización de un plan de estudios dinámicamente estructurado en experiencias de aprendizaje.

- Dimensión de actividades, ejercicios y procedimientos de la mediación como formas pedagógicas de la autoorganización del conocimiento.

- Conciencia de la importancia de un lenguaje liberador en el acto educativo, esto contribuye notablemente a satisfacer las demandas de los aprendientes, además la expresión de sentimientos y pensamientos debe llevarse a cabo en un ambiente de respeto, solidaridad y trabajo conjunto.

- El conocimiento se expande y amplía cuando hay una cálida relación social, entendimiento y libertad para expresarse. 


\section{Aprendizaje social}

Este ambiente holístico propicia el desarrollo de interacciones sociales. El conocimiento se expande y amplía cuando hay una cálida relación social, entendimiento y libertad para expresarse. En grupo se crean las interacciones sociales de una manera afable, mostrando respeto, solidaridad y tolerancia. La sinergia lleva a las personas a un estado de transformación personal, de colaboración, de apreciación de intereses, de identificación con el sentir del otro y de apoyo emocional. Se aprende a construir conocimiento en grupo, a pensar y reflexionar para encontrar acciones que se puedan poner en marcha en la búsqueda de la verdad. De hecho, las experiencias grupales de aprendizaje centran la atención en la vida porque mejoran la autoestima, hacen la vida más plena, desarrollan la creatividad, las actividades mentales, la memoria, la autonomía, la autosuficiencia, el equilibrio mental y emocional, la identidad, la capacidad de aprender, y aumentan los niveles de expectativas.

Dentro de este nuevo paradigma, el aprendizaje se vuelve vida. Las experiencias de aprendizaje ayudan a transformar la realidad, la cual se convierte en una atmósfera de nuevas ideas y amor por el aprendizaje. Cada experiencia es un despertar de la conciencia espiritual para aprender a ser y descubrir lo que lo hace feliz en un contexto cultural y social que le da la oportunidad de integrarse exitosamente a la sociedad moderna y transformarla. En el nuevo paradigma, las experiencias de aprendizaje tienen el siguiente propósito, «...formar un sujeto para la vida. Un sujeto capaz de vivir y discernir estructuras sociales, regímenes de propiedad y formas de cálculo económico en función de la vida real» ${ }^{21}$. Cuando el proceso de aprendizaje ocurre en un ambiente ameno o plácido. la persona se abre a la curiosidad y a la investigación.

\footnotetext{
21 Franz Hinkelammert y Henry Mora, Hacia una economía para la vida (San José: Departamento Ecuménico de Investigaciones, 2005) 28.
} 
Este ambiente holístico debe incorporar las experiencias propuestas por Maturana, para quien la base biológica del amor es el soporte de la formación humana de la educación; él propone que en la educación, fundamento para desarrollar destrezas emocionales en el educar, reine el respeto, el amor, la consideración, la cooperación, la responsabilidad, la convivencia y la legitimidad del otro ${ }^{22}$.

La educación ha de crear espacios para la convivencia feliz, sin temores, sin miedos o incertidumbres; propiciar libertad con el objetivo de que el aprendiente se cree y se auto cree con confianza en que el aprendizaje va a ser significativo. Además esta nueva educación, rescata valores realmente importantes en los cuales «tanto los individuos como la propia dinámica de los conjuntos organizacionales tienen que impregnarse de un nuevo humanismo» ${ }^{23}$.

\section{Conclusión}

Con el fin de concienciar a los aprendientes acerca de los cambios que se dan en nuestra realidad, quienes educan han de promover la sensibilidad en el aprendiente para que desarrolle su capacidad de asombro y sea capaz de generar cambios importantes, sobre todo en cuanto a su interés por el aprendizaje de idiomas. En las aulas, la construcción de conocimiento se trasforma en una actividad que genera placer en el proceso de aprendizaje y de descubrimiento.

Este ambiente debe ser holístico. las actividades no se pueden ver aisladas, sino en forma integral con todo lo existente en el entorno; así, se despierta un sentimiento entremezclado de valores como libertad, humildad, responsabilidad. amor, respeto por los demás. De csta manera, se crea conciencia sobre la necesidad de apreciar integralmente el cuerpo, los sentimientos y el espíritu. Cada experiencia de aprendizaje es una oportunidad para fomentar los valores y

Humberto Maturana. Transformación en la comivencia (Santiago: Dolmen Ediciones. 1999).

Assmann. 62. 
principios universales, pues vivir es aprender. Este principio es esencial para el desarrollo de una segunda lengua.

Ya hemos iniciado esta opción hacia una educación más atrayente dentro de las aulas de estudio. Somos los entes de cambio con espíritu de autorreflexión que generan oportunidades para aprender, construir y crear nuevas ideas. La liberación de la imaginación abre brechas para crear soluciones a los conflictos y buscar nuevas alternativas educativas que faciliten estos procesos pedagógicos. 